\title{
Numerical modelling of flow pattern for high swirling flows
}

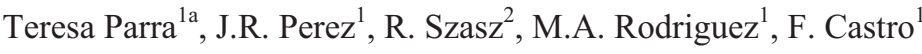 \\ ${ }^{1}$ University of Valladolid, Spain. \\ ${ }^{2}$ Lund University, Sweden.
}

\begin{abstract}
This work focuses on the interaction of two coaxial swirling jets. High swirl burners are suitable for lean flames and produce low emissions. Computational Fluid Dynamics has been used to study the isothermal behaviour of two confined jets whose setup and operating conditions are those of the benchmark of Roback and Johnson. Numerical model is a Total Variation Diminishing and PISO is used to pressure velocity coupling. Transient analysis let identify the non-axisymmetric region of reverse flow. The center of instantaneous azimuthal velocities is not located in the axis of the chamber. The temporal sampling evidences this center spins around the axis of the device forming the precessing vortex core (PVC) whose Strouhal numbers are more than two for Swirl numbers of one. Influence of swirl number evidences strong swirl numbers are precursor of large vortex breakdown. Influence of conical diffusers evidence the reduction of secondary flows associated to boundary layer separation.
\end{abstract}

\section{Introduction}

An analysis was carried out to study numerically the interaction of two isothermal coaxial jets, the annual one with azimuthal velocity component. Large Eddy Simulation methodology to reproduce the case of Roback and Johnson [1], see figure 1.

The swirl generator was modified to produce different swirl numbers. It is composed by 8 flat blades with a certain pitch angle. Swirl number is defined as the ratio of azimuthal and axial velocities. The angle of the trailing edges is $62^{\circ}$ for swirl number 1.2 and $45^{\circ}$ for swirl number 0.6 , respectively.

Bearing in mind the annular jet with strong swirl number, a vortex breakdown with bubble shape appears in the axis of the device named Inner Recirculation Zone (IRZ). Besides, the sudden expansion with a ratio of 2 in diameter between nozzle exits and the test chamber produces the boundary layer separation with the corresponding torus shape recirculation identified as Outer Recirculation Zone (ORZ). The zone between the inner and the outer recirculation zones is the shear layer.

\section{Numerical Model}

An implicit LES model was used, this model uses mesh size as filter width so no sub-grid model is required. It is the numerical error who plays the role of dissipative part of the stress tensor in the submesh.
The van Driest's wall damping function is responsible to cancel turbulent viscosity near the wall [2]. Mesh prerequisites are $\Delta \mathrm{y}^{+}=1$ and uniform hexahedral mesh.

The resolution scheme used is a Total Variation Diminishing (TVD) with coefficients looking for good precision. PISO is the pressure- velocity coupling used [3]. Besides, multigrid resolution improves the performance towards the full convergence.

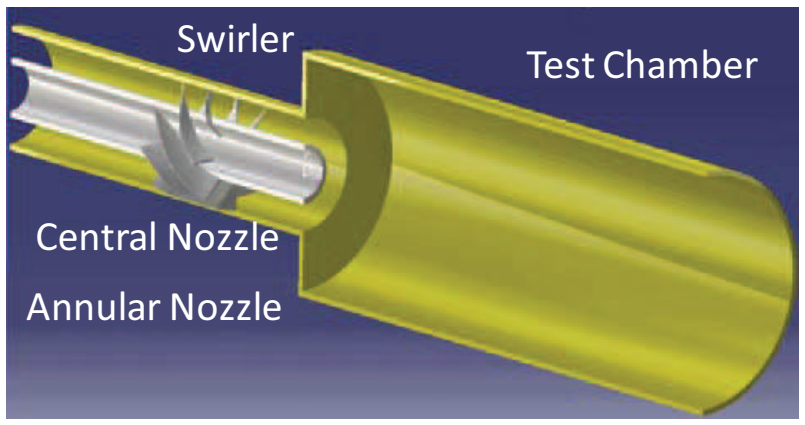

Figure 1. Set up of the numerical model.

The Reynolds number is around 2400 based on bulk velocity at the test chamber with a Kolmogorov scale around $0.17 \mathrm{~mm}$. The transitional turbulence regime is simulated using a mesh with above 10 million grid points with a resolution lower than $1 \mathrm{~mm}$ per cell.

The boundary conditions and the geometrical details are summarized in table 1.

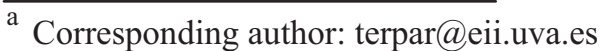


Table 1. Summary of geometrical details and the inlet conditions for the nozzles.

\begin{tabular}{lccc}
\hline \multicolumn{1}{c}{ Magnitude } & $\begin{array}{c}\text { Central } \\
\text { jet }\end{array}$ & $\begin{array}{c}\text { Annular } \\
\text { inlet }\end{array}$ & $\begin{array}{c}\text { Test } \\
\text { Chamber }\end{array}$ \\
\hline Diameter $(\mathrm{m})$ & $0-0.025$ & $0.0306-0.059$ & 0.122 \\
Velocity $(\mathrm{m} / \mathrm{s})$ & 0.66 & 1.52 & \\
Intensity of & 12 & 7.5 & \\
turbulence $(\%)$ & & & \\
Density $\left(\mathrm{kg} / \mathrm{m}^{3}\right)$ & & 1.225 & \\
Viscosity & & $1.789410^{-5}$ & \\
$(\mathrm{~kg} /(\mathrm{ms}))$ & & & \\
\hline
\end{tabular}

\section{Flow Pattern for Swirl Number 1.2}

Figures 2 show the longitudinal behaviour of the mean and instantaneous velocity field, as well as the variance to gain an idea of the velocity standard deviation around its time averaged value.

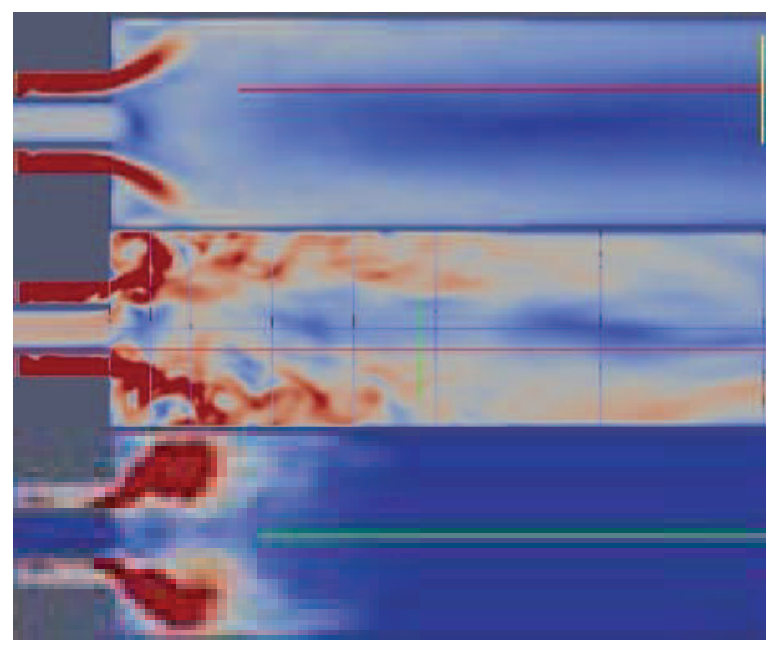

Figure 2. Longitudinal contour of (top) Mean velocity, (middle) Instantaneous velocity (bottom) Variance of the resolved scales. Swirl number 1.2.

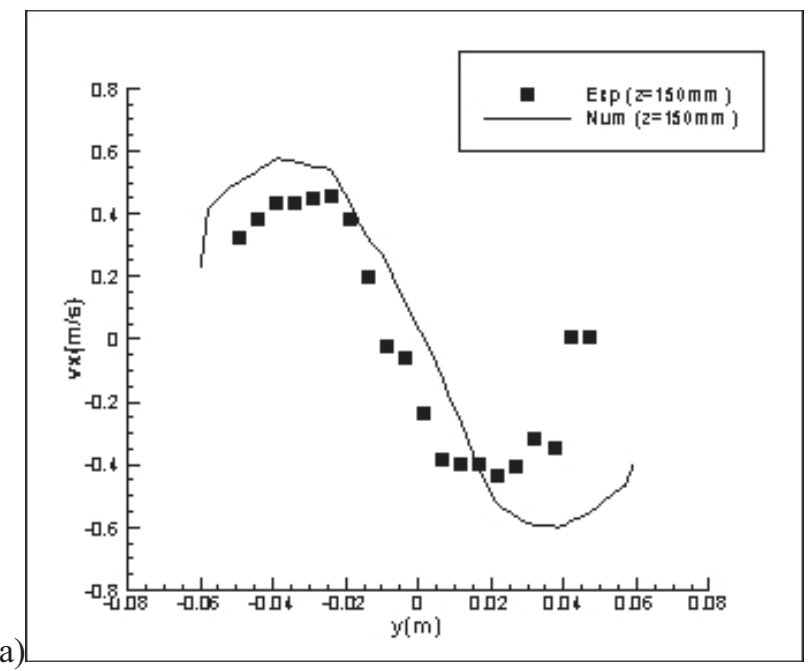

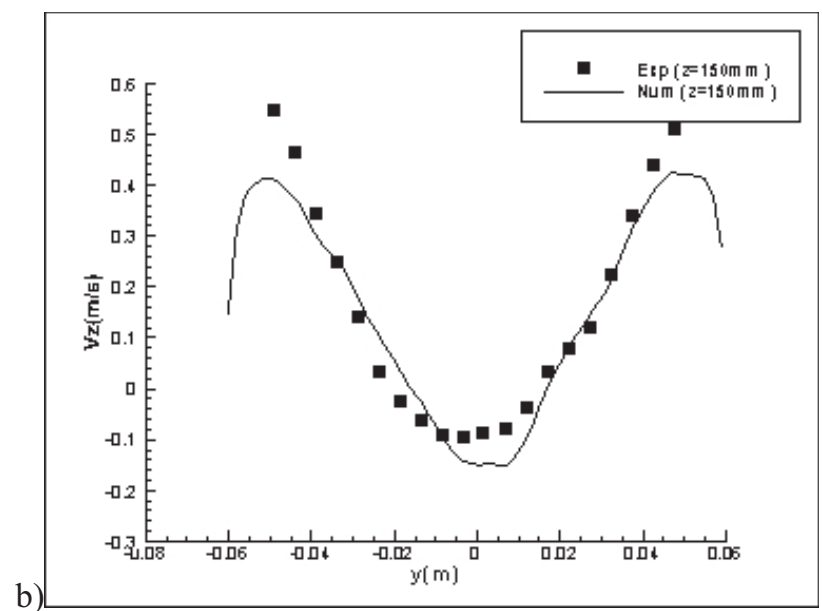

Figure 3. Radial profiles of averaged velocity components (a) azimuthal velocity, (b) axial velocity.

Figure 3 contrast experimental results from Roback and Johnson [1] with the predictions of the numerical model for both axial and tangential components of the averaged velocity. The linear tendency of the tangencial velocity evidences a forced vortex spinning around the axis of the chamber. The negative values of axial velocity in the center of the chamber corresponds with the reverse flow of the IRZ.

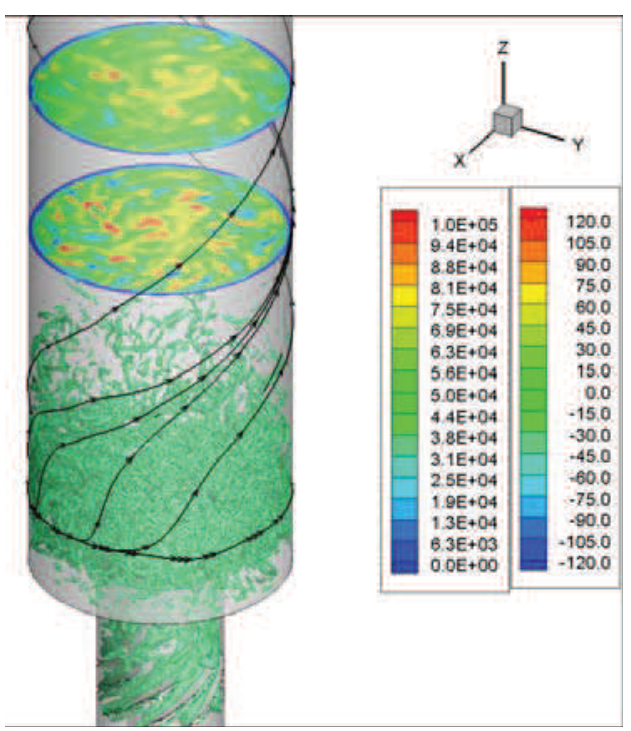

Figure 4. Iso-surfaces of $Q=30000$. Slices at axial positions $\mathrm{z}=$ $17.5 \mathrm{~mm}$ and $25 \mathrm{~mm}$ with axial vorticity ranging from -120 to $120 \mathrm{~s}-1$.

When LES is used, a large number of vortices appear that are difficult to identify using criteria as vorticity maximum or minimum pressure. It is preferable to use lamda2 negative or $\mathrm{Q}$ positive as criteria for identifying the core of the vortices. These parameters are calculated as a function of strain and rotation tensors, $\mathrm{S}$ and $\Omega$, respectively. Lamda2 is the second the eigenvalue of the tensor $S^{2}+\Omega^{2}$ whereas $Q$ is defined by equation (1)

$$
\mathrm{Q}=-\frac{1}{2}\left(\mathrm{~S}_{\mathrm{ij}} \mathrm{S}_{\mathrm{ij}}-\Omega_{\mathrm{ij}} \Omega_{\mathrm{ij}}\right) \text {. }
$$


Figure 4 shows the iso- surface of $\mathrm{Q}$ for a positive value as well as axial vorticity in two transversal slices.

\section{Transient Analyses}

Transient analysis let identify the non-axisymmetric region of reverse flow. The centre of instantaneous azimuthal velocities is not located in the axis of the chamber. The temporal sampling evidences this centre spins around the axis of the device forming the precessing vortex core (PVC) whose Strouhal numbers are more than two for Swirl numbers of one [4-5]

Figures 5 and 6 depict the velocity vectors on transversal sections located at 0.04, 0.4 and 1.22 times the chamber diameter from the nozzles' exit. The color contours corresponds with the axial velocity. Their negative values represent the reverse flow. The black dot is the geometric center of the system.

Figure 5 top is very near the exit; hence it is possible to identify the central jet whose diameter was $25 \mathrm{~mm}$ surrounded by the annular swirling jet with an external diameter of $59 \mathrm{~mm}$. Also it is clear the outer recirculation zone (ORZ) due to the sudden expansion.

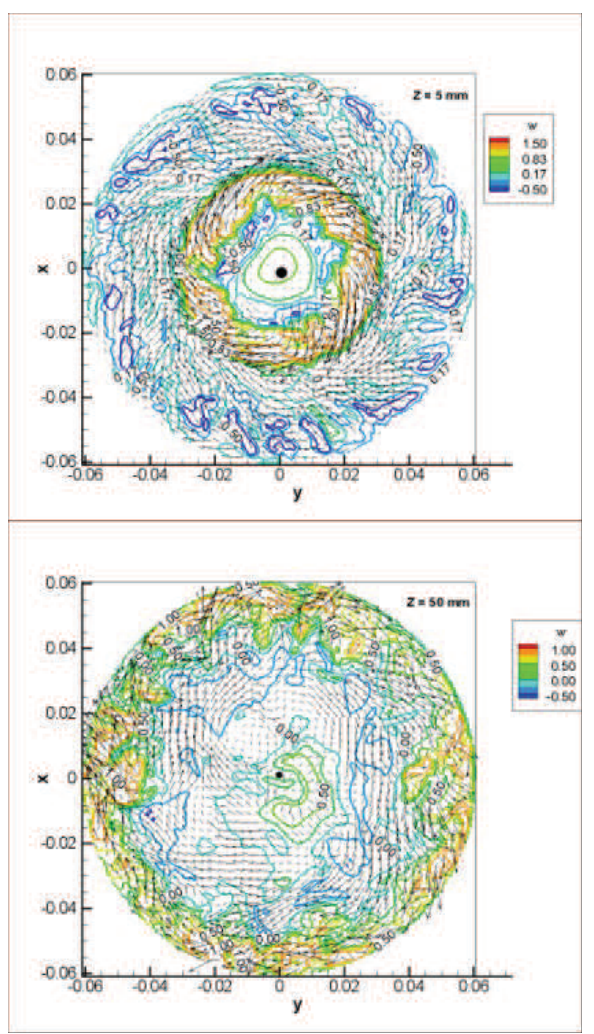

Figure 5. Instantaneous velocity field in the transversal sections: (top) $\mathrm{Z}=0.005 \mathrm{~m}$ and (bottom) $0.05 \mathrm{~m}$. Axial velocity contours and velocity vectors.

Figure 5 bottom is the transversal section at $50 \mathrm{~mm}$ from the nozzles' exit. The outer recirculation zone is not present any more but an inner recirculation zone (IRZ) appears. This slice let identify the precessing vortex core (PVC), the spinning around the axis of the chamber.
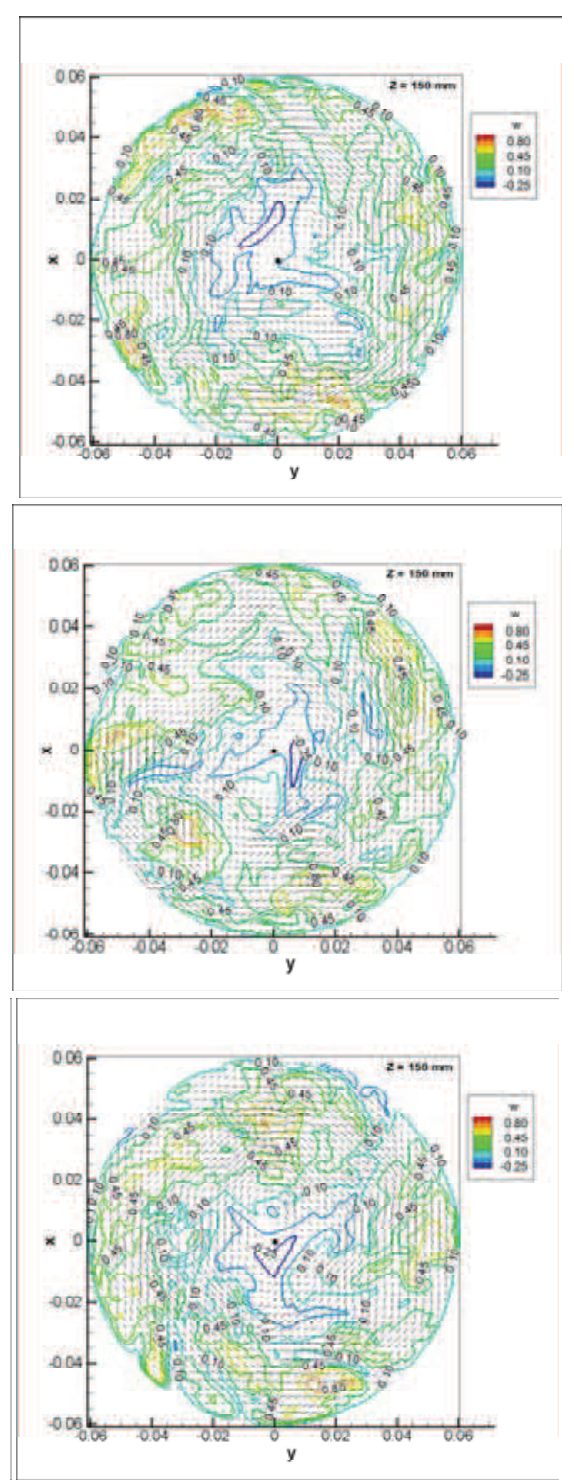

Figure 6. Instantaneous velocity field in the transversal section $\mathrm{Z}=0.15 \mathrm{~m}$. Contours correspond with axial velocity over velocity vectors.

The non-axisymmetric behavior evidences a zone with high axial velocity opposite to the reverse flow. The PVC is where the azimuthal velocity is null, here it is located in the IRZ but not in the geometrical center.

Figures 6 show three instantaneous velocity fields over one period of the PVC for the transversal section located at 1.22 times the diameter of the chamber. It is possible to evidence the spinning of the core around the geometrical center.

The different instantaneous trajectories of PVC are depicted in figure 7 which have been capture as the location with null instantaneous azimuthal velocity. It is clear the core spins around the axis of the chamber. The frequency of spinning corresponds with a Strouhal number around 2.83 calculated based on the chamber diameter and its bulk velocity. 


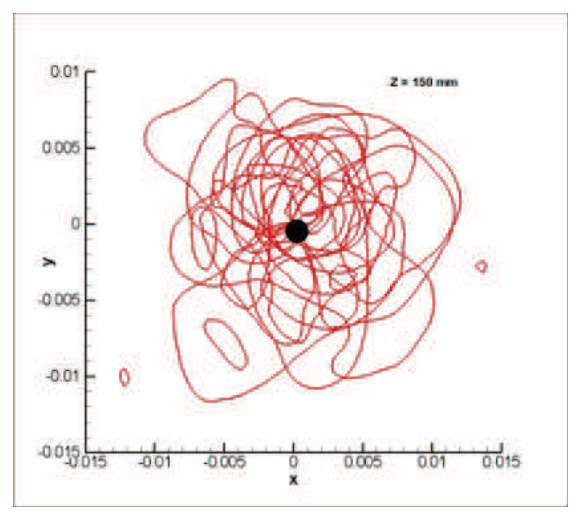

Figure 7. Different instantaneous iso- azimuthal velocity zero in the transversal section $\mathrm{Z}=0.15 \mathrm{~m}$

\section{Influence of Swirl number}

This section is devoted to contrast strong and weak swirl numbers. Despite the simple geometrical configuration, the flow has complex features.
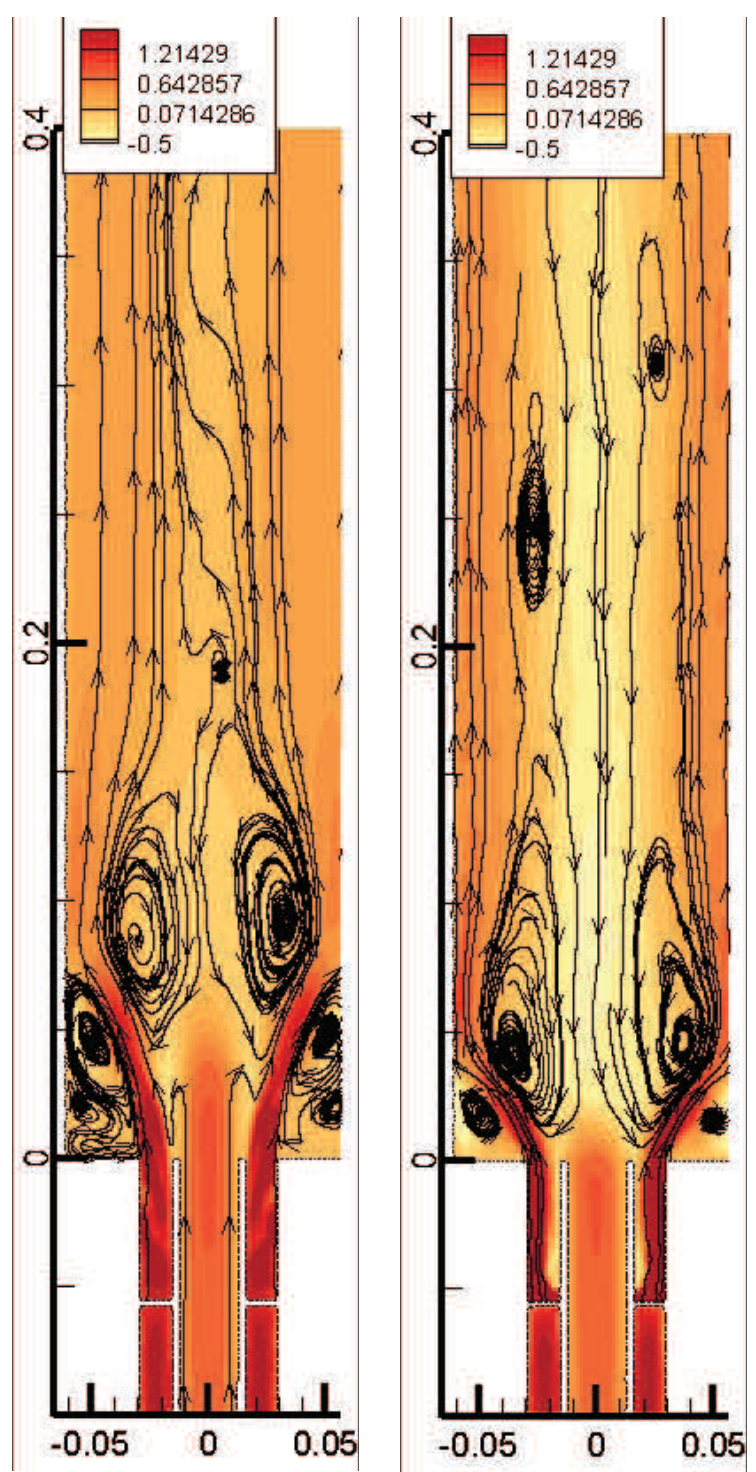

Figure 8. Averaged Axial Velocities in longitudinal slice as well as projected stream lines. Right) Swirl 1.2, Left) Swirl 0.6.
Strong swirl numbers produce high radial pressure gradients [6]. Hence, the ORZ is confined while IRZ suffer large radial expansion, see figure 8 right. Whereas, low swirl numbers produce the lead stagnation point to be farer from exit and the ORZ is larger, see figure 8 left. ORZ is characterized by counter rotating vortex rings increasing head losses. These structures are the CouetteTaylor instabilities.

\section{Influence of Conical Diffuser}

This section is devoted to analyze the influence of conical diffusers. Figures 9 indicate diffusers with angles lower than 140 prevent the formation of ORZ for swirl 1.2.

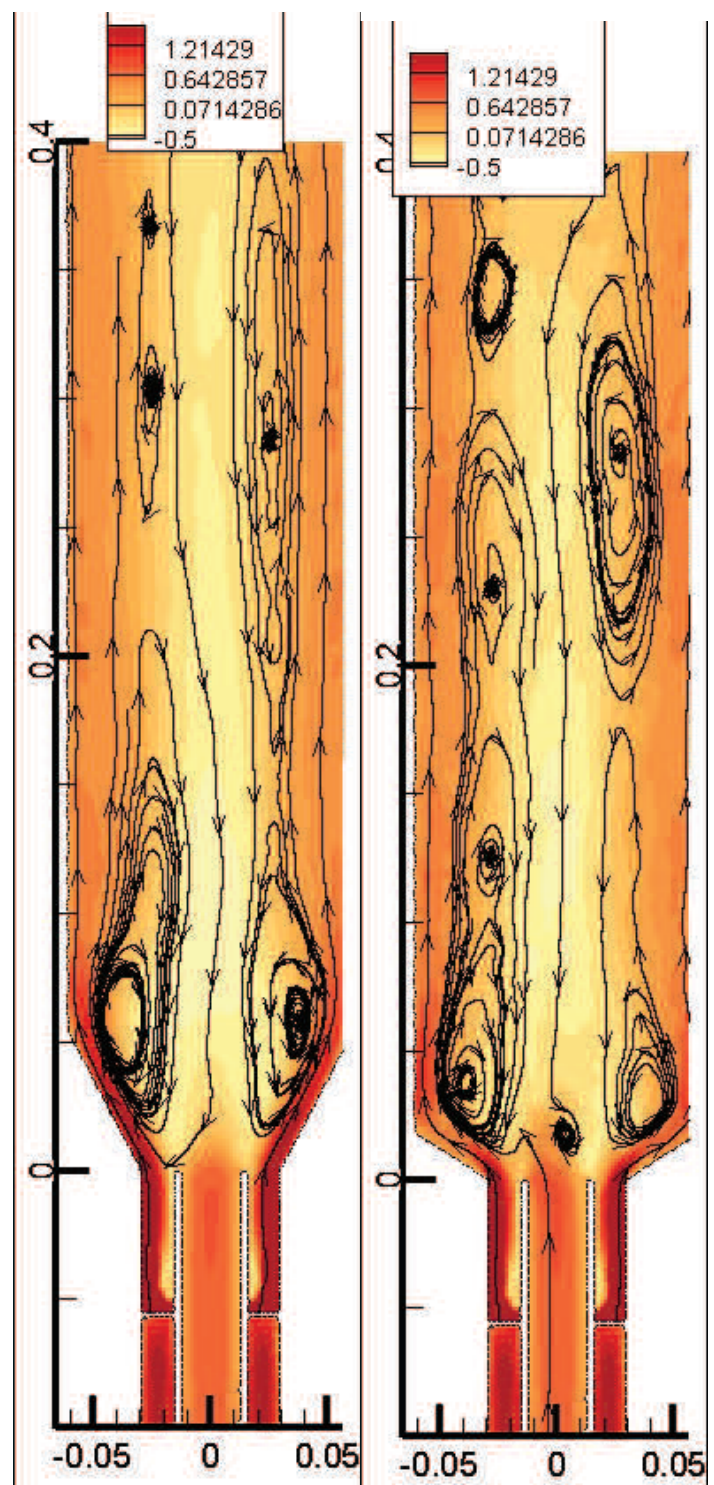

Figure 9. Averaged Axial Velocities in longitudinal slice for Swirl 1.2 with conical diffuser of angles $60^{\circ}$ (left), $120^{\circ}$ (right).

Figure 10 shows the performance of diffusers for swirl number 0.6. Diffusers with angle $120^{\circ}$ prevent the formation of counter rotating vortex rings whereas a diffuser lower than $60^{\circ}$ is necessary to avoid the presence of ORZ. 

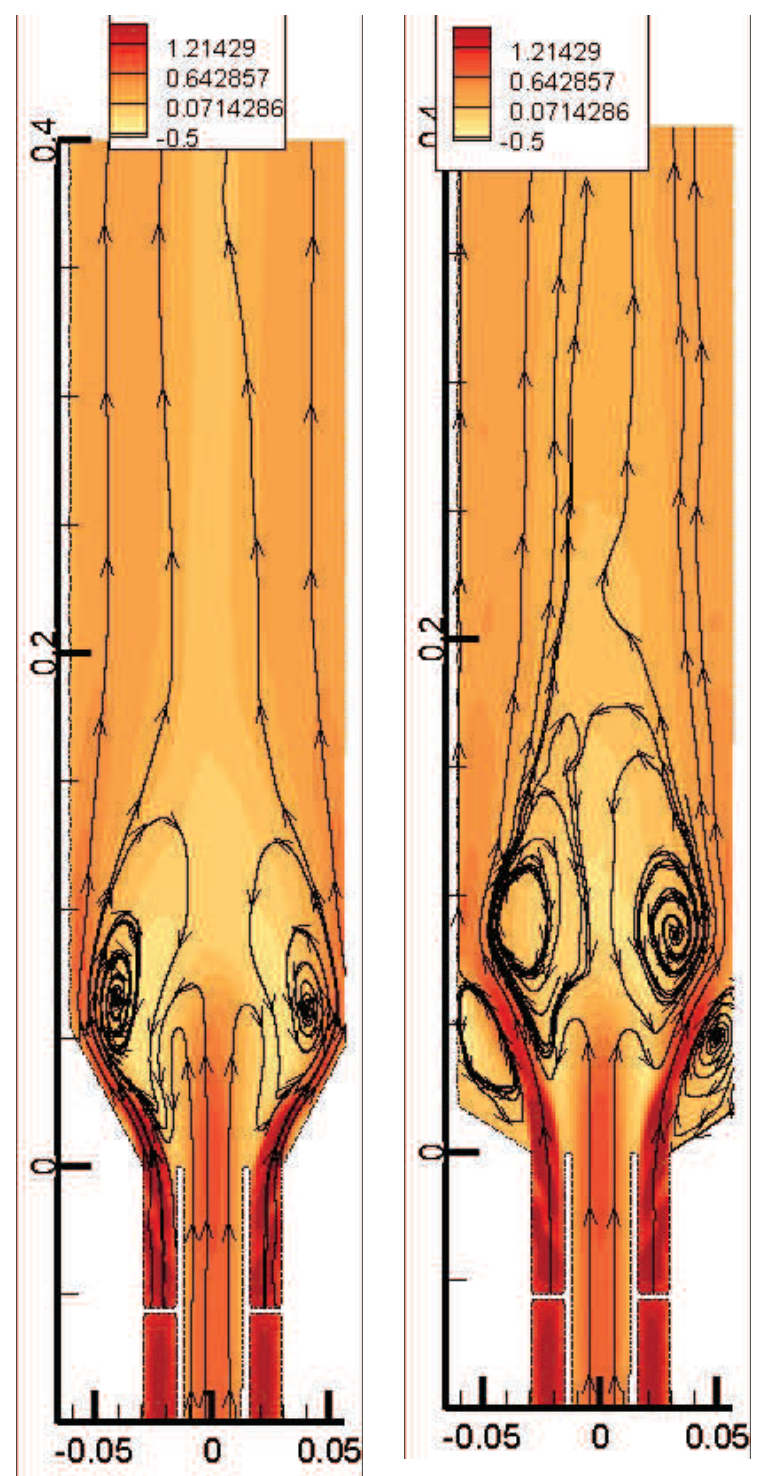

Figure 10. Averaged Axial Velocities in longitudinal slice for Swirl 0.6 with conical diffuser of angles $60^{\circ}$ (left), $120^{\circ}$ (right).

\section{Conclusions}

The interaction of two isothermal confined swirling jets was model. Implicit LES is a very challenging approach to model flows.

Transient analysis let identity non-axisymmetric behavior of the inner recirculation zones as well as the location and frequency of the PVC for a swirl number of 1.1

Averaged fluid field let analyse the flow patterns. Swirl numbers 1.2 and 0.6 were contrasted. Strong swirl numbers produce larger IRZ and smaller ORZ than low swirl numbers.

A conical diffuser prevents or reduces the ORZ.

\section{Acknowledgment}

The author thankfully acknowledges the Spanish Ministry of Science and Innovation for the financial resources in the framework of the project reference ENE2011-25468.

We acknowledge PRACE for awarding us access to resource Curie-GENCI@CEA based in France and MareNostrum@BSC based in Spain. Ref. 2010PA1766

\section{References}

1. R. Roback, B.V. Johnson. NASA CR-168252 (1983)

2. T. Parra, V. Vuorinen, R. Perez, R. Szasz and F. Castro. International Journal of Energy and Environmental Engineering 5:85 (2014)

3. V. Vuorinen, M. Larmi, P. Schlatter, L. Fuchs, and B.J. Boersma Computers \& Fluids. 70, 195-205 (2012).

4. N. Syred. Progress in Energy and Combustion Science 32 93-161 (2006)

5. T. Parra, R. Z. Szasz, C. Duwig, R. Pérez, V. Mendoza, and F. Castro International Journal of Mechanical Engineering Vol:7 No:9, pp 742-745 (2013)

6. T. Parra-Santos; JR. Pérez-Domínguez; VM. Mendoza-García; RZ. Szasz; F. Castro; AN Gutkowski; Engineering Computations. Accepted 23.07.2014. 